\title{
Patient and physician perspectives on engaging in palliative and healthcare trials: a qualitative descriptive study
}

Valeria Cardenas ${ }^{1 *}$, Anna Rahman ${ }^{1}$, Jenna Giulioni ${ }^{1,2}$, Alexis Coulourides Kogan ${ }^{1,3}$ and Susan Enguidanos ${ }^{1}$

\begin{abstract}
Background: Researchers are encountering increasing challenges in recruiting participants for palliative and healthcare research. This paper aims to understand challenges to and methods for engaging physicians and seriously ill patients and their caregivers in research studies.

Methods: Between October 2019 to July 2020, we conducted qualitative interviews with 25 patients, proxies, and caregivers participants who were eligible for a randomized controlled trial of home-based palliative care and 31 physicians from participating accountable care organizations. Using thematic analysis, we analyzed participants' responses to identify concepts and key ideas within the text. From these initial concepts, core themes around barriers to research and preferred research recruitment approaches were generated.
\end{abstract}

Results: Themes from patient and caregiver interviews included time constraints, privacy concerns, lack of research familiarity, disconnect with research institution, self-perceived health status, and concerns with study randomization. Physician-identified barriers focused on time constraints and study randomization. Patient and caregiver recommendations for study recruitment included in-person recruitment, recruitment at healthcare providers' offices, recruitment via mail, additional study information, and frequent calls. Physician recommendations were related to placement of flyers at clinics, financial incentives, and formal events.

Conclusions: Findings demonstrated that although patients and caregivers prefer that their physicians recruit them for health-related research studies, physicians identified time constraints as a consistent barrier to research involvement.

Keywords: Palliative care, Qualitative study, Referral, Primary care physicians, Caregivers, Recruitment

\section{Background}

Healthcare research is critical to improving treatment, reducing disease and symptoms, and reducing avoidable costs of health care [1], yet studies have found increasing difficulty in engaging participants in research trials [2]. Additionally, recruitment and retention are often the most challenging aspect of conducting research

\footnotetext{
*Correspondence: vlcarden@usc.edu

${ }^{1}$ Leonard Davis School of Gerontology, University of Southern California,

3715 McClintock Ave., GER 208B, Los Angeles, CA 90089, USA

Full list of author information is available at the end of the article
}

[3-5], let alone when patients with serious illness are targeted $[6,7]$. This is concerning given that the need for improved care among seriously ill patient has been well documented $[8,9]$ and remains a focus of research efforts nationally [10].

Palliative care research, with its focus on improving quality of life for patients with serious illness, faces significant challenges in appropriate patient identification and enrollment in research trials. For example, investigators of an early Palliative Care Research Cooperative trial who conducted interviews with study site personnel 
identified three primary challenges to recruitment related to patients and physicians: (1) locating trial-eligible patients; (2) severity of patient illness; and (3) physician/ caregiver gatekeeping over patients [11]. Additionally, insufficient patient recruitment also has been identified as a challenge in the majority of National Cancer Institute funded studies of palliative care [12]; further highlighting the importance of understanding challenges and facilitators to patient recruitment.

Involving physicians in research also has been identified as a challenge [13-15]. Although primary care physicians (PCPs) report positive experiences in conducting research [16], PCPs face financial and time constraints in their work, preventing them from engaging in research [17]. Additionally, healthcare providers may fear burdening their patients and may lack clear understanding of the research topic or what will be asked of participants, thereby serving as gatekeepers to research participation among their patients [18]. This gatekeeping behavior has made it difficult for researchers to recruit seriously ill patients into studies, including palliative care trials [19-21].

In a wide-reaching qualitative synthesis review of facilitators and barriers related to patient and caregiver involvement in palliative care research, Chambers and colleagues identified several themes that ranged from definition of research and palliative care to organization cultural and diversity [21]. Notably researchers identified a paucity of rigorous studies on patient and caregiver barriers and facilitators to palliative care research and called for more rigorous investigations and studies that included patients and caregivers in the design and implementation.

Furthermore, despite more than a decade of research on palliative care practices, there remain significant gaps in research to advance this field. Researchers analyzed more than 600 published studies on palliative care to identify priorities for future research. Among the most frequently identified areas in need of more research were the perspectives and needs of patients, relatives, and providers [22].

While previous studies have documented barriers encountered in palliative care research, few have studied these barriers from patient, caregiver, and physician perspectives who had been approached to participate in a randomized controlled trial (RCT). Additionally, few studies focus on identifying strategies to improve research trial engagement among physicians and seriously ill patients and their family members. To fill this gap, we conducted a qualitative descriptive study to identify challenges to engaging patients, proxies, caregivers, and physicians in research and their preferred strategies methods for research recruitment. We also aimed to understand the relationship between the patient and physician perspectives and elicit specific recommendations to better engage both patients and physicians in palliative care research, a unique contribution of this investigation.

\section{Methods}

To achieve this aim, we conducted individual interviews with primary care physicians, patients, and caregivers who previously had been approached to be part of a RCT [23]. The original RCT aimed to compare the effectiveness of home-based palliative care and primary care enhanced by added training in palliative care principles among seriously ill patients with congestive heart failure, chronic obstructive pulmonary disease, or advanced cancer and their caregivers [23], but was terminated due to under enrollment [24]. The RCT and this qualitative descriptive study were individually approved by the Institutional Review Board of the University of Southern California. Our study report follows the Consolidated Criteria for Reporting Qualitative Studies (COREQ) guidelines [25].

\section{Recruitment}

From October 2019 to July 2020, we used purposive sampling to recruit PCPs from two accountable care organizations (ACOs) and patients (and their caregivers) who had been previously approached to participate in an RCT. We purposively selected both those who had agreed to be part of the research study as well as those who declined. Patients and caregivers were initially mailed a letter explaining the current study and invited to participate in a telephone interview. We followed the letter with a telephone call. When a patient lacked capacity to consent, the research assistant interviewed the proxy. We used various strategies to recruit PCPs for the current study; PCPs were invited to participate through faxed and mailed study flyers, telephone calls to their office, and holiday cards. In a couple of cases, we visited their office to invite them to participate. All participants were invited to participate in a $30-\mathrm{min}$ interview.

\section{Interview protocol}

The study team, comprised of experts in palliative care and research, developed a semi-structured protocol to elicit information about PCPs, patients', and caregivers' perceptions on research participation, including their perceived barriers to participation and their suggestions for overcoming these challenges. The interview protocol was reviewed by patient and caregivers study advisors as well as by an outside researcher familiar with our study. After the first 
interview, the study protocol was slightly modified for ease of administration.

The protocol included questions inquiring about patient, proxy, and caregiver concern with research participation and their preferred method of study recruitment. PCPs were asked what they perceived as barriers to providing patient referrals to research studies and suggestions for overcoming these barriers. See Additional file 1 for interview questions. Patients, proxies, and caregivers received a \$50 (USD) gift card for their participation. Physicians initially received a $\$ 150$ (USD) gift card, which was later increased to $\$ 300$ (USD) to improve participation rates. Patient, proxy, and caregiver interviews were conducted by a female study manager with a Master of Public Health degree and PCP interviews by a doctoral trained female (A.C.K.) with more than 10 years of research experience, including qualitative interviewing. The interviewers disclosed their role In the research study to study participants. All interviews were conducted via telephone with the exception of one PCP interview taking place in-person. All interviews were audio-recorded, transcribed verbatim, and lasted between 10 and $54 \mathrm{~min}$.

At the conclusion of each interview, demographic information was collected from all participants. Patient, proxy, and caregiver information included age, gender, ethnicity, marital status, education, work status, and current medical conditions. PCPs reported their age, gender, ethnicity, country of birth, and number of years working in healthcare and in their current position.

\section{Analysis}

Interview transcripts were transferred to an Excel sheet by trained research assistants and were analyzed by three researchers (V.C., J.G., Y.Z.). Using thematic analysis [26], researchers familiarized themselves with the data by reading the transcripts and writing down initial concepts and key ideas. The team convened to discuss this initial list of ideas and consolidated related concepts before arriving at a single list of themes. The same researchers reread and independently coded the transcripts using this list of themes, while comparing and linking themes throughout the process. The researchers met again and final codes were compared and discussed until 100\% agreement was reached between coders. In the final step, researchers extracted examples for each theme. We conducted this process separately for both the PCP interviews and the patient/proxy/caregiver interviews. Thematic saturation was reached after the 10th interview for patients/proxy/caregivers and after the 14th interview for PCPs, however we continued to code all transcripts. Final themes were shared with our study investigator team and our patient and caregiver stakeholders for feedback. Stakeholder feedback did not change any of the final themes.

\section{Results}

From October 2019 to July 2020, we interviewed 56 participants: 17 patients, eight caregivers/proxies, and 31 PCPs. Of the patients, proxies, and caregivers, we recruited 15 from among 109 RCT-eligible participants who refused to participate in the earlier study and 10 from the 28 participants who enrolled in the RCT. Among potential patients and caregivers, five had died, five were unreachable, three refused to participate, and three declined for other reasons. Of the PCPs, we contacted 198 physicians of which 31 responded to our invitation to participate in our study.

\section{Participant demographic characteristics}

Table 1 reports the demographic characteristics of the participating patients, caregivers, proxies, and PCPs. Among the 17 patient participants, the majority were female (70.6\%), white (82.4\%), and married (70.6\%). More than half (53\%) had attained a college degree or higher, and $64.7 \%$ were retired. Patients reported currently having one or more medical conditions. Six patients (35.3\%) had enrolled in the larger RCT, while 11 (64.7\%) had declined to participate.

Among the eight caregivers and proxies, 50\% were male, white, married, college graduates, and retired. Caregivers/proxies reported having fewer medical conditions than patients. Half the caregivers/proxies had a family member who had enrolled in the larger RCT and the other half had declined to participate. All proxies interviewed were family members, with one identifying as a caregiver as well. Additional participant demographics are reported in Table 1.

Of the 31 physician participants, 17 (54.8\%) were male and $14(45.2 \%)$ were female. Nearly half $(45.2 \%)$ were white, 9 (29\%) were Asian, six (19.4\%) identified as other, and two (6.5\%) were Latino/Hispanic. Ages ranged from 39 to 75 years $(\mathrm{M}=57 ; \mathrm{SD}=9.17)$. More than half $(58.1 \%)$ of the physicians were foreign-born. Most (77.4\%) reported working in healthcare for more than 20 years.

\section{Themes}

From the data, we identified six themes related to barriers to healthcare research and eight recommendations for study recruitment. Themes are presented below and in Table 2 .

\section{Barriers to healthcare research \\ Time}

Patients, proxies, caregivers, and PCPs identified time constraints as a barrier to research participation. Patients, proxies, and caregivers shared that their time 
Table 1 Participant Characteristics $(N=56)$

\begin{tabular}{|c|c|c|c|c|}
\hline & $\begin{array}{l}\text { All } \\
(\mathrm{N}=56)\end{array}$ & $\begin{array}{l}\text { Patients Only } \\
(N=17)\end{array}$ & $\begin{array}{l}\text { Caregivers/Proxy Only } \\
(\mathrm{N}=8)\end{array}$ & $\begin{array}{l}\text { Physicians Only } \\
(N=31)\end{array}$ \\
\hline Characteristics & $\mathrm{n}(\%)$ & & & \\
\hline Age (mean $\pm S D)$ & $58.91 \pm 10.74$ & $64.06 \pm 9.83$ & $55.88 \pm 15.23$ & $56.87 \pm 9.17$ \\
\hline \multicolumn{5}{|l|}{ Gender } \\
\hline Male & $26(46.4 \%)$ & $5(29.4 \%)$ & $4(50 \%)$ & $17(54.8 \%)$ \\
\hline Female & $30(53.6 \%)$ & $12(70.6 \%)$ & $4(50 \%)$ & $14(45.2 \%)$ \\
\hline \multicolumn{5}{|l|}{ Ethnicity } \\
\hline Black/African American & $2(3.6 \%)$ & $1(5.9 \%)$ & $1(12.5 \%)$ & $0(0 \%)$ \\
\hline White/Caucasian & $32(57.1 \%)$ & $14(82.4 \%)$ & $4(50 \%)$ & $14(45.2 \%)$ \\
\hline Hispanic/Latino & $4(7.1 \%)$ & $1(5.9 \%)$ & $1(12.5 \%)$ & $2(6.5 \%)$ \\
\hline Asian & $11(19.6 \%)$ & $1(5.9 \%)$ & $1(12.5 \%)$ & $9(29 \%)$ \\
\hline Other & $7(12.5 \%)$ & $0(0 \%)$ & $1(12.5 \%)$ & $6(19.4 \%)$ \\
\hline \multicolumn{5}{|l|}{ US Born } \\
\hline Yes & $18(58.1 \%)$ & & & $18(58.1 \%)$ \\
\hline No & $13(41.9 \%)$ & & & $13(41.9 \%)$ \\
\hline \multicolumn{5}{|l|}{ Marital Status $^{\mathrm{a}}$} \\
\hline Single & $3(12 \%)$ & $2(11.8 \%)$ & $1(12.5 \%)$ & \\
\hline Married/Living with a partner & $16(64 \%)$ & $12(70.6 \%)$ & $4(50 \%)$ & \\
\hline Widowed & $2(8 \%)$ & $0(0 \%)$ & $2(25 \%)$ & \\
\hline Divorced & $4(16 \%)$ & $3(17.7 \%)$ & $1(12.5 \%)$ & \\
\hline \multicolumn{5}{|l|}{ Education $^{\mathrm{a}}$} \\
\hline High School Graduate & $3(12 \%)$ & $2(11.8 \%)$ & $1(12.5 \%)$ & \\
\hline Some College & $9(36 \%)$ & $6(35.3 \%)$ & $3(37.5 \%)$ & \\
\hline College Graduate & $11(44 \%)$ & $7(41.2 \%)$ & $4(50 \%)$ & \\
\hline Post Graduate & $2(8 \%)$ & $2(11.8 \%)$ & $0(0 \%)$ & \\
\hline \multicolumn{5}{|l|}{ Work Status } \\
\hline Work full-time & $6(24 \%)$ & $3(17.7 \%)$ & $3(37.5 \%)$ & \\
\hline Unemployed & $4(16 \%)$ & $3(17.7 \%)$ & $1(12.5 \%)$ & \\
\hline Retired & $15(60 \%)$ & $11(64.7 \%)$ & $4(50 \%)$ & \\
\hline \multicolumn{5}{|l|}{ Years working in health care ${ }^{b}$} \\
\hline 11 to 15 & $5(16.1 \%)$ & & & $5(16.1 \%)$ \\
\hline 16 to 20 & $2(6.5 \%)$ & & & $2(6.5 \%)$ \\
\hline Over to 20 years & $24(77.4 \%)$ & & & $24(77.4 \%)$ \\
\hline \multicolumn{5}{|c|}{ Years working in certain position ${ }^{b}$} \\
\hline Less than a year & $0(0 \%)$ & & & $0(0 \%)$ \\
\hline 1 to 5 & $4(12.9 \%)$ & & & $4(12.9 \%)$ \\
\hline 6 to 10 & $4(12.9 \%)$ & & & $4(12.9 \%)$ \\
\hline 11 to 15 & $4(12.9 \%)$ & & & $4(12.9 \%)$ \\
\hline 16 to 20 & $7(22.6 \%)$ & & & $7(22.6 \%)$ \\
\hline Over to 20 years & $12(38.7 \%)$ & & & $12(38.7 \%)$ \\
\hline \multicolumn{5}{|l|}{ Medical Condition ${ }^{\mathrm{a}}$} \\
\hline Cancer & $5(20 \%)$ & $4(23.5 \%)$ & $1(12.5 \%)$ & \\
\hline COPD & $4(16 \%)$ & $4(23.5 \%)$ & $0(0 \%)$ & \\
\hline Heart Disease & $8(32 \%)$ & $8(47.1 \%)$ & $0(0 \%)$ & \\
\hline Liver Disease & $2(8 \%)$ & $2(11.8 \%)$ & $0(0 \%)$ & \\
\hline Diabetes & $8(32 \%)$ & $8(47.1 \%)$ & $0(0 \%)$ & \\
\hline Arthritis & $9(36 \%)$ & $7(41.2 \%)$ & $2(25 \%)$ & \\
\hline \multicolumn{5}{|l|}{ Study Status } \\
\hline Enrolled & $17(30.4 \%)$ & $6(35.3 \%)$ & $4(50 \%)$ & $7(22.6 \%)$ \\
\hline Declined & $39(69.6 \%)$ & $11(64.7 \%)$ & $4(50 \%)$ & $24(77.4 \%)$ \\
\hline
\end{tabular}

Footnotes:

a Patients \& Caregivers $(N=25)$

b Physicians Only $(\mathrm{N}=31)$ 


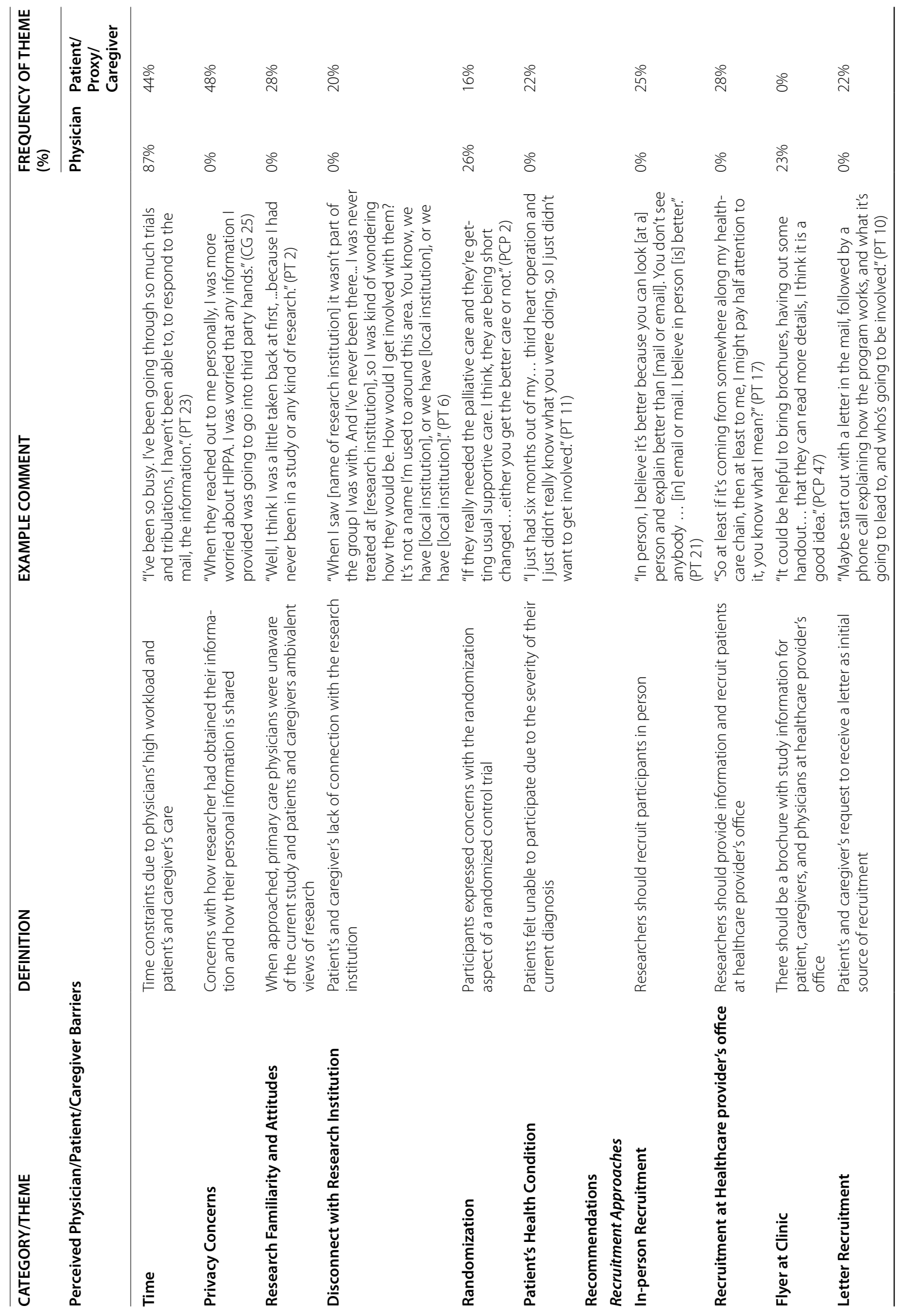




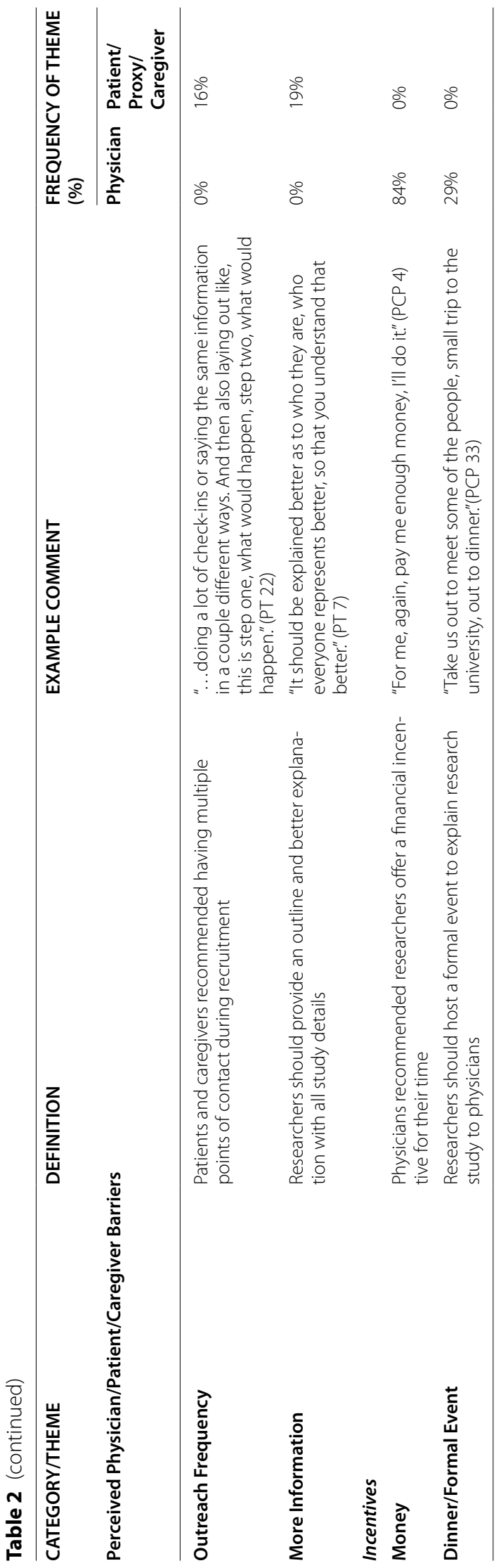


was constrained by taking care of their health or the health of their loved one.

"I felt like there was so much going on and I was concerned about adding another something I need to do." (PT 22)

Nearly all PCPs mentioned being unable or unwilling to participate in research due to their busy schedules. One physician (PCP 1) stated that:

"The main challenge... is that when you're in the middle of a really busy clinical day, it's really hard to have one extra thing that you need to do."

\section{Privacy concerns}

Several patients, proxies, and caregivers mentioned that they were wary about how the research team had obtained their information and whether their information would be provided to a third party. One patient (PT 17) shared her concerns about potential fraud and the legitimacy of the recruitment call. The lack of notification of the study prior to the call contributed to her distrust and concern about a potential telephone scam:

"I don't know why I was being contacted, it was basically just someone calling me out of the blue, and it could have been just something fake, [someone] trying to get my personal information."

\section{Disconnect with the research institution}

Also contributing to the distrust in the research recruitment call was that patients stated they had no connection with the research institution and questioned how a non-local institution would conduct research in their town. One patient (PT 7) described the geographic distance between the research institute and his residence as a source of disconnect:

"You guys are out in [location of research institution], I was in [hometown], so I don't know how you would have had somebody in my [hometown].

\section{Research familiarity and attitudes}

Patients, proxies, and caregivers shared their negative perceptions about research and the novelty of participating in a research study. Some mentioned how the word "research" made them uncomfortable, not wanting to enroll because they had no prior experience with research or simply disliked interviews. Some patients had discussed the study with their doctors, and their doctor who had no knowledge of the research study.
"To be honest, I've never been in one [research study], it is probably something that I'm not comfortable in... I don't [see] myself involved in those kinds of things." (PT 6)

\section{Randomization}

PCPs, patients, proxies, and caregivers were concerned about the randomization process and the potential to be assigned to a group that did not receive palliative care. Similarly, several physicians expressed concern that patients might not receive needed services due to randomization.

"It becomes a little concerning...in my mind, they need the [service] and if [the patient] is going to get it or not, becomes more like an ethical thing." (PCP 61)

A caregiver enrolled in the previous RCT said her only concern with the study would have been "not being chosen for the in-home care." (CG 2).

\section{Patients' health condition}

Many patients said they could not participate in the earlier RCT because they were too sick. Patients discussed having gone through multiple hospitalizations, being too ill, and not being able to 'deal with it all'. As one patient (PT 7) said:

"When you're really sick, it's something hard to just concentrate on answering random multiple-choice questions... because you're not really sure where your own health is going at the time."

\section{Recommendations \\ Recruitment approaches}

In-person recruitment Many participants recommended that research recruitment occur in-person. Some believed in-person recruitment could facilitate participant understanding of the study and help researchers understand patients' current health status. One caregiver (CG 13) said:

"...there is nothing better than to do [research recruitment] personally, no telephone, no filling out brochures... It is much easier to meet with a person for 30 minutes to an hour."

Recruitment at healthcare Provider's office More specifically, several patients, proxies, and caregivers recommended that recruitment occur at the physician's office or prior to being discharged from the hospital. As one caregiver mentioned (CG 15): 
"I'm thinking when [the patient] has a doctor's appointment-if you would be able to go to a doctor's appointment and while they were waiting for their doctor's appointment [they could be approached to participate in a study] ...or before they left the hospital."

Flyers at clinics PCPs suggested that researchers leave flyers for patients at their clinics and an outline of the research project for physicians. A physician (PCP 43) proposed:

"If I have an outline of the research projects that you are conducting that will help me basically, keep your [study in] mind for when I see the patients."

Letter recruitment Some participants recommended that they receive study information by mail before receiving the first recruitment phone call. A patient (PT 8) said:

"A letter in the mail is way better than just a phone call because most people won't answer the phone because they don't know the [phone] number."

Outreach Frequency Several patients, proxies, and caregivers recommended the research team contact them multiple times to make sure the message comes across appropriately. One patient emphasized the importance of multiple recruitment calls:

"...making that second and third call back... to let them know what you are doing. And eventually they're going to see what you're trying to tell them. (PT 6)

More information Many patients, proxies, and caregivers said they needed a clearer explanation of the study and of palliative care; they recommended that the research team provide multiple sources of information. An enrolled participant from the previous RCT (\#10) suggested:

"[having]...an outline of how it's going to be conducted and what it's going to lead to: 'We will be calling you every month to ask questions', and 'We will be referring you to other local organizations for one-on-one help?"'

\section{Incentives}

Money While financial incentives were not mentioned by patients, proxies, and caregivers, nearly all PCPs said a financial incentive would encourage physician research participation. One physician (PCP 2) said he would need "... enough of a reimbursement to make it worth my while."

Formal event Several PCPs suggested that the research teams host a formal event to explain their study. A physician (PCP 47) mentioned:

"... offering dinners at nice restaurants and then saying these opportunities we'd like to get you engaged [in]..."

\section{Discussion}

This study identified challenges related to conducting and participating in research as perceived by PCPs, patients, and caregivers/proxies. Through this new understanding of the relationship between the patient, proxy, caregiver, and physician perspectives, this study provides a better understanding of how researchers could work with physicians to build participant trust in research by providing a "warm handoff" during recruitment. Additionally, our findings highlight the need for researchers to address physician's challenges, namely in ensuring adequate time and/or incentives are provided for physician inclusion in research trials.

In particular, nearly all PCPs in this study identified time as a major challenge in participating in research, a finding widely supported by previous research $[13,16$, $17,21,27]$. Studies have shown that despite physician awareness of potential long-term benefits of participating in research, healthcare providers are inundated with the immediate demands of patient care; thus, patient referrals to research studies become a lower priority [14].

Patients, proxies, and caregivers felt their time was constrained by their poor health and their medical care needs. In a multi-site RCT of patients with end-stage renal disease, nearly half (47\%) had refused participation due to the severity of their disease [28]. Similarly, a palliative care clinical trial found the most commonly cited reason for refusing study participation was that the patient felt too sick [29].

Participants also identified challenges that were specific to the RCT's design. Several patients, proxies, and caregivers were concerned about randomization and not being able to select the palliative care intervention 
themselves. Often patients view randomization as a loss of control and prefer that their doctor select their treatment [30, 31]. Those who had no previous research involvement were wary of what a research study would entail. With this lack of research familiarity came privacy concerns: participants were nervous about how their information was obtained and with whom it would be shared.

Patients also were concerned about their physician's lack of awareness of the RCT. Lack of awareness of ongoing clinical trials among physicians was identified as a major challenge to patient recruitment in five academic medical centers in the United States (U.S.) [32]. PCPs' lack of awareness and their attitudes towards research can influence patient exposure to research participation.

A couple of the barriers we identified related to issues of trust in relation to the organization conducting study outreach. From the patient, proxy, and caregiver perspective, the research institute did not have a clear connection with their healthcare provider, which may have heightened concerns around privacy. Additionally, "cold calling" without prior notification of study recruitment (via letter, through their physician, etc) also heightened their sense of distrust in the legitimacy of the research call.

\section{Recruitment approaches}

Patients, proxies, and caregivers suggested multiple strategies and approaches to recruiting participants for research studies. These included recruitment via mail, in-person, or at healthcare providers' offices. These different approaches have all demonstrated some success. Face-to-face recruitment yielded the highest response rates in a study of three health networks in the U.S. [33]. Others have suggested that physician involvement and recruitment at their office is the most important and effective recruitment strategy $[34,35]$. Physician recommendations and referrals to research studies have been a strong factor to participation [36]. While our participants preferred their PCP to introduce the study, PCPs in our study discussed the many time constraints that would prevent them from actively recruiting patients for research. Instead, they suggested recruitment efforts that would not impinge on their time, such as placement of fliers in their clinic.

In addition, patients, proxies, and caregivers suggested sending a recruitment letter prior to making the first phone call, which has been an integral part of study recruitment [37]. When receiving the first call, participants requested numerous phone calls to grab their attention, while others disputed this approach earlier.

\section{Incentives}

Nearly all PCPs discussed how a financial incentive could encourage them to participate in research. Lack of financial incentives has been identified as a barrier to participating in research with PCPs [16]. Studies have found that some physicians believe monetary compensation demonstrates that the physician's time is valued, [27] and that this incentive also can increase a physician's likelihood to participate in research [38].

Interestingly, incentives did not arise in the interviews conducted among patients, proxies, and caregivers. Anecdotally, just one respondent discussed financial incentives and mentioned that healthcare was more important than a monetary incentive. While a systematic literature review on health research study participation found that provision of monetary incentives increased patient participation between 4 to $23 \%$ [39], little is known about the usefulness of monetary incentives in palliative care research or research among seriously ill patients.

\section{Strengths and limitations}

To our knowledge, this is the first study to investigate barriers and recommendations to overcoming these barriers from the perspective of PCPs, patients, proxies, and caregivers after the conclusion of a previous research trial. This study was conducted with a purposive sample of RCT-eligible patients, caregivers, and physicians contracted with an ACO in the U.S. Responses may have been influenced by their experiences with the larger RCT's recruitment efforts and their level of participation in the RCT. The participants interviewed in this study may have declined participation in the larger RCT but were willing to take part in this qualitative descriptive study. Therefore, the results do not represent the perspective of potential participants who decline all research participation, another strength of this study in that perspectives include those consenting and declining a larger research trial. Additionally, this study focuses exclusively on stakeholder perceptions of research recruitment, and does not include strategies for reducing participant attrition, another challenge when conducting research with populations with serious illness [40, 41].

Future studies are needed to compare effectiveness of recruitment strategies to determine best practices for engaging physicians and seriously ill patients in research.

\section{Conclusion}

This study found that patients, proxies, and caregivers may be cautious when approached for palliative care research participation, and therefore prefer inperson recruitment for research trials as well as being recruited by their physician. However, we also found 
that physicians have limited time to participate in studies. These findings underscore the importance of offering incentives as a strategy to increase physician research involvement.

\section{Abbreviations}

PCP: Primary care physician; RCT: Randomized controlled trial; COREQ: Consolidated Criteria for Reporting Qualitative Studies; ACOs: Accountable care organizations; PT: Patient; U.S.: United States.

\section{Supplementary Information}

The online version contains supplementary material available at https://doi. org/10.1186/s12904-021-00856-6.

\section{Additional file 1.}

\section{Acknowledgements}

The authors want to thank the physicians, patients, and caregivers who participated in this study.

\section{Authors' contributions}

S.E. and A.R. conceptualized the design of this research with support from A.C.K. A.C.K. collected the interview data. V.C. and J.G. undertook the data analyses. V.C. led the write-up of the manuscript with all the authors providing critical input and revision of the manuscript drafts. The author(s) read and approved the final manuscript.

\section{Funding}

The authors disclosed receipt of the following financial support for the research, authorship, and/or publication of this article: V.C. is supported by the National Institute On Aging of the National Institutes of Health under Award Number T32AG000037. The content is solely the responsibility of the authors and does not necessarily represent the official views of the National Institutes of Health. This study is funded through a Patient-Centered Outcomes Research Institute (PCORI) Award IHS-1602-34070. The opinions presented in this manuscript are solely the responsibility of the authors and do not necessarily represent the views of the PCORI, its Board of Governors, or Methodology Committee.

\section{Availability of data and materials}

The datasets used and analyzed during the current study are available from the corresponding author on reasonable request.

\section{Declarations}

\section{Ethics approval and consent to participate}

The study was reviewed and approved by the University of Southern California Institutional Review Board (UP-17-00257). All methods were performed in accordance with these guidelines and regulations. Under this approval, informed verbal consent was obtained from all participants. Following the approved recruitment procedures, all participants first were sent written information on the study. They were then contacted by a research assistant who again reviewed the study, answered questions, and obtained verbal permission to participate in the study and to audio-tape the interview.

\section{Consent for publication}

Not applicable.

\section{Competing interests}

The authors declare that they have no competing interests.

\section{Author details}

${ }^{1}$ Leonard Davis School of Gerontology, University of Southern California, 3715 McClintock Ave., GER 208B, Los Angeles, CA 90089, USA. ${ }^{2}$ Division of Biokinesiology and Physical Therapy, University of Southern California, Los Angeles, CA 90033, USA. ${ }^{3}$ Keck School of Medicine, Department of Family Medicine and Geriatrics, University of Southern California, Alhambra, CA 91803, USA.

Received: 4 May 2021 Accepted: 28 September 2021

Published online: 14 October 2021

\section{References}

1. Nass SJ, Levit LA, Gostin LO. The HIPAA privacy rule. In: Gostin LO, Levit LA, Nass SJ, editors. Beyond the HIPAA privacy rule: enhancing privacy, improving health through research. Washington (DC): National Academies Press (US); 2009.

2. Galea S, Tracy M. Participation rates in epidemiologic studies. Ann Epidemiol. 2007;17(9):643-53.

3. James AS, Daley CM, Engelman K, Greiner KA, Ellerbeck E. Process evaluation of recruitment for a cancer screening trial in primary care. Health Promot Pract. 2011;12(5):696-703.

4. Kadam RA, Borde SU, Madas SA, Salvi SS, Limaye SS. Challenges in recruitment and retention of clinical trial subjects. Perspect Clin Res. 2016;7(3):137.

5. Adams M, Caffrey L, McKevitt C. Barriers and opportunities for enhancing patient recruitment and retention in clinical research: findings from an interview study in an NHS academic health science Centre. Health ResPolicy Syst. 2015;13(1):1-9.

6. Ferguson GT, Beck B, Clerisme-Beaty E, Liu D, Thomashow BM, Wise RA, et al. Recruiting patients after hospital discharge for acute exacerbation of COPD: challenges and lessons learned. Chronic Obstructive Pulmonary Diseases: J COPD Foundation. 2017;4(4):265.

7. Stafford L, Sinclair M, Newman L, Rauch P, Barton M, Gilham L, et al. Why did we fail? Challenges recruiting parents with cancer into a psychoeducational support program. Psycho-oncology. 2019;28(12):2425-8.

8. Singer AE, Meeker D, Teno JM, Lynn J, Lunney JR, Lorenz KA. Symptom trends in the last year of life from 1998 to 2010: a cohort study. Ann Intern Med. 2015;162(3):175-83.

9. Connors AF, Dawson NV, Desbiens NA, Fulkerson WJ, Goldman L, Knaus WA, et al. A controlled trial to improve care for seriously ill hospitalized patients: the study to understand prognoses and preferences for outcomes and risks of treatments (SUPPORT). Jama. 1995;274(20):1591-8.

10. Cassel CK, Field MJ, editors. Approaching death: improving care at the end of life: National Academies Press; 1997.

11. Hanson LC, Bull J, Wessell K, Massie L, Bennett RE, Kutner JS, et al. Strategies to support recruitment of patients with life-limiting illness for research: the palliative care research cooperative group. J Pain Symptom Manage. 2014;48(6):1021-30.

12. Rinck GC, Van den Bos GA, Kleijnen J, de Haes HJ, Schadé E, Veenhof $\mathrm{CH}$. Methodologic issues in effectiveness research on palliative cancer care: a systematic review. J Clin Oncol. 1997;15(4):1697-707.

13. Ross S, Grant A, Counsell C, Gillespie W, Russell I, Prescott R. Barriers to participation in randomised controlled trials: a systematic review. J Clin Epidemiol. 1999:52(12):1143-56.

14. Steinhauser KE, Clipp EC, Hays JC, Olsen M, Arnold R, Christakis NA, et al. Identifying, recruiting, and retaining seriously-ill patients and their caregivers in longitudinal research. Palliat Med. 2006;20(8):745-54.

15. Kleiderman E, Avard D, Black L, Diaz Z, Rousseau C, Knoppers BM. Recruiting terminally ill patients into non-therapeutic oncology studies: views of health professionals. BMC Med Ethics. 2012;13(1):1-2.

16. Khalaf AJ, Aljowder Al, Buhamaid MJ, Alansari MF, Jassim GA. Attitudes and barriers towards conducting research amongst primary care physicians in Bahrain: a cross-sectional study. BMC Fam Pract. 2019;20(1):1-5.

17. Iloh GP, Amadi AN, Iro OK, Agboola SM, Aguocha GU, Chukwuonye ME. Attitude, practice orientation, benefits and barriers towards health research and publications among medical practitioners in Abia state, Nigeria: a cross-sectional study. Niger J Clin Pract. 2020;23(2):129.

18. McHugh KR, Swamy GK, Hernandez AF. Engaging patients throughout the health system: a landscape analysis of cold-call policies and recommendations for future policy change. J Clini TransI Sci. 2018;2(6):384-92. 
19. Ewing G, Rogers M, Barclay S, McCabe J, Martin A, Todd C. Recruiting patients into a primary care based study of palliative care: why is it so difficult? Palliat Med. 2004;18(5):452-9.

20. Stone PC, Gwilliam B, Keeley V, Todd C, Kelly LC, Barclay S. Factors affecting recruitment to an observational multicentre palliative care study. BMJ Support Palliat Care. 2013;3(3):318-23.

21. Chambers E, Gardiner C, Thompson J, Seymour J. Patient and carer involvement in palliative care research: an integrative qualitative evidence synthesis review. Palliat Med. 2019;33(8):969-84.

22. Riffin C, Pillemer K, Chen EK, Warmington M, Adelman RD, Reid MC. Identifying key priorities for future palliative care research using an innovative analytic approach. Am J Public Health. 2015;105(1):e15-21.

23. Enguidanos S, Rahman A, Fields T, Mack W, Brumley R, Rabow M, Mert M. Expanding access to home-based palliative care: a randomized controlled trial protocol. J Palliat Med 2019;22(S1):S-58.

24. Enguidanos S, Rahman A. Early termination of a palliative care trial: perspectives of multiple stakeholders on barriers to palliative care and research. J Palliat Med 2021;0(0)1-7.

25. Tong A, Sainsbury P, Craig J. Consolidated criteria for reporting qualitative research (COREQ): a 32-item checklist for interviews and focus groups. International J Qual Health Care. 2007;19(6):349-57.

26. Braun V, Clarke V. Using thematic analysis in psychology. Qual Res Psychol. 2006;3(2):77-101.

27. Albers $L L$, Sedler KD. Clinician perspectives on participation in research. J Midwifery Womens Health. 2004;49(1):47-50.

28. Brickey JA, Flannery M, Cuthel AM, Cho J, Grudzen CR. Barriers to recruitment into a randomized controlled trial comparing two modes of emergency department-initiated palliative care. Research Square. [Preprint]. 2020.

29. LeBlanc TW, Lodato JE, Currow DC, Abernethy AP. Overcoming recruitment challenges in palliative care clinical trials. J Oncol Pract. 2013;9(6):277-82

30. Jenkins $V$, Fallowfield $L$. Reasons for accepting or declining to participate in randomized clinical trials for cancer therapy. Br J Cancer. 2000;82(11):1783-8.

31. Sheridan R, Martin-Kerry J, Hudson J, Parker A, Bower P, Knapp P. Why do patients take part in research? An overview of systematic reviews of psychosocial barriers and facilitators. Trials. 2020;21(1):1-8.
32. Tanner A, Kim SH, Friedman DB, Foster C, Bergeron CD. Barriers to medical research participation as perceived by clinical trial investigators: communicating with rural and African American communities. J Health Commun. 2015;20(1):88-96.

33. Heerman WJ, Jackson N, Roumie CL, Harris PA, Rosenbloom ST, Pulley J, et al. Recruitment methods for survey research: findings from the midsouth clinical data research network. Contemp Clin Trials. 2017;62:50-5.

34. Ngune I, Jiwa M, Dadich A, Lotriet J, Sriram D. Effective recruitment strategies in primary care research: a systematic review. Qual Prim Care. 2012;20(2):115-23.

35. Kinney AY, Richards C, Vernon SW, Vogel VG. The effect of physician recommendation on enrollment in the breast Cancer chemoprevention trial. Prev Med. 1998;27(5):713-9.

36. Avis NE, Smith KW, Link CL, Hortobagyi GN, Rivera E. Factors associated with participation in breast cancer treatment clinical trials. J Clin Oncol. 2006;24(12):1860-7.

37. Herber OR, Schnepp W, Rieger MA. Recruitment rates and reasons for community physicians' non-participation in an interdisciplinary intervention study on leg ulceration. BMC Med Res Methodol. 2009;9(1):1-8.

38. Young RA, Fulda KG, Suzuki S, Hahn KA, Espinoza AM, Marshall JD, et al. The influence of research compensation options on practice-based research network (PBRN) physician participation: a North Texas (NorTex) PBRN study. J Am Board Fam Med. 2011;24(5):562-8.

39. Huynh L, Johns B, Liu SH, Vedula SS, Li T, Puhan MA. Cost-effectiveness of health research study participant recruitment strategies: a systematic review. Clin Trials. 2014;11(5):576-83.

40. Dobratz MC. Issues and dilemmas in conducting research with vulnerable home hospice participants. J Nurs Scholarsh. 2003;35(4):371-6.

41. Jordhøy MS, Kaasa S, Fayers P, Underland G, Ahlner-Elmqvist M. Challenges in palliative care research; recruitment, attrition and compliance: experience from a randomized controlled trial. Palliat Med. 1999;13(4):299-310.

\section{Publisher's Note}

Springer Nature remains neutral with regard to jurisdictional claims in published maps and institutional affiliations.
Ready to submit your research? Choose BMC and benefit from:

- fast, convenient online submission

- thorough peer review by experienced researchers in your field

- rapid publication on acceptance

- support for research data, including large and complex data types

- gold Open Access which fosters wider collaboration and increased citations

- maximum visibility for your research: over $100 \mathrm{M}$ website views per year

At BMC, research is always in progress.

Learn more biomedcentral.com/submissions 